\title{
Atendimento domiciliar odontológico ao idoso em tempos de pandemia por COVID-19
}

\author{
Dental home care for the elderly in pandemic times by COVID-19
}

Atención dental domiciliaria para ancianos em tienpos de pandemia por COVID-19

Gisseli Matioli ${ }^{1 *}$, Maria Antonia Fernandes Nabarro Oliveira Benati ${ }^{1}$, Mirian Cristina Ribeiro dos Santos ${ }^{1}$.

\begin{abstract}
RESUMO
Objetivo: Discorrer sobre a forma de atendimento odontológico em domicílio em tempos de pandemia causado pelo COVID-19 visando diminuir o risco de contaminação pelo SARS-CoV-2, onde nesta modalidade o idoso, permanece no ambiente "livre de contaminação" e só recebe o profissional, para tratamentos de urgência e emergência, com os devidos cuidados. Revisão bibliográfica: Apurou-se com o presente estudo que devido a pandemia causada pelo SARS-CoV-2, desde dezembro de 2019 são repensadas novas formas de atendimento domiciliar ao idoso. A modalidade de atendimento é praticada em todo o mundo, e no Brasil vem se destacando ao longo dos anos. O intuito é evitar as formas mais comuns de contágio de COVID 19 com protocolo de biossegurança ao entrar e sair da residência, priorizando atendimentos de urgência e emergência, proporcionando melhor qualidade de vida ao idoso. Considerações finais: $O$ cuidado em relação à contaminação por COVID-19 deve ser observado pelos profissionais da saúde e o atendimento domiciliar ao idoso é uma proposta que precisa ser visualizada como uma medida eficaz preventiva multiprofissional, principalmente nos casos de emergência ou mesmo em casos onde o idoso não possa comparecer em consultório odontológico visando, sua perspectiva e qualidade de vida.
\end{abstract}

Palavras-chave: Home care, Odontologia, COVID-19.

\begin{abstract}
Objective: To discuss the form of dental care at home in times of pandemic caused by COVID-19 in order to reduce the risk of contamination by SARS-CoV2, where in this modality the elderly person remains in the "contamination-free" environment and only receives the professional, for urgent and emergency treatments, with due care. Bibliographic review: It was found with the present study that due to the pandemic caused by SARS-CoV-2, new forms of home care for the elderly have been rethought since December 2019. The service modality is practiced worldwide, and in Brazil it has stood out over the years. The aim is to avoid the most common forms of contagion of COVID 19 with a biosafety protocol when entering and leaving the residence, prioritizing urgent and emergency care, providing better quality of life for the elderly. Final considerations: Care in relation to COVID-19 contamination must be observed by health professionals and home care for the elderly is a proposal that needs to be viewed as an effective multiprofessional preventive measure, especially in emergency cases or even in cases where the elderly cannot attend a dental office aiming at their perspective and quality of life.
\end{abstract}

Key words: Home care, Dentistry, COVID-19.

\footnotetext{
1 Faculdade Estácio São Paulo (FSP), Rolim de Moura - RO.

*E-mail: gisseli.farm@gmail.com
}

SUBMETIDO EM: 11/2019

ACEITO EM: 1/2021

PUBLICADO EM: 4/2021 


\section{RESUMEN}

Objetivo: Discutir la forma de cuidado dental en el hogar en tiempos de pandemia por COVID-19 con el fin de reducir el riesgo de contaminación por SARS-CoV-2, donde en esta modalidad el anciano permanece en el ambiente "libre de contaminación" y Recibe únicamente el profesional, para tratamientos de urgencia y emergencia, con la debida atención. Revisión bibliográfica: Se encontró con el presente estudio que debido a la pandemia causada por el SARS-CoV-2, se han replanteado nuevas formas de atención domiciliaria para los ancianos desde diciembre de 2019. La modalidad de servicio se practica a nivel mundial y en Brasil se ha destacado a lo largo de los años. El objetivo es evitar las formas más habituales de contagio de COVID 19 con un protocolo de bioseguridad a la entrada y salida de la residencia, priorizando la atención urgente y de emergencia, proporcionando una mejor calidad de vida a las personas mayores. Consideraciones finales: La atención en relación a la contaminación por COVID-19 debe ser observada por los profesionales de la salud y la atención domiciliaria al anciano es una propuesta que debe ser vista como una medida preventiva multiprofesional eficaz, especialmente en casos de emergencia o incluso en los casos en que los ancianos no pueden atender un consultorio dental orientado a su perspectiva y calidad de vida.

Palabras clave: Cuidados en el hogar, Odontología, COVID-19.

\section{INTRODUÇÃO}

O novo coronavírus (SARS-CoV-2), surgiu na China no fim de 2019 e em pouco tempo tornou-se uma pandemia, decretado pela Organização Mundial de Saúde (OMS) em março de 2020. A infecção pelo SARSCoV-2, denominada de COVID-19 tem uma elevada capacidade de infecção sendo passível de transmissão tanto de forma direta como indireta por espirro, tosse, saliva e fluidos (PEREIRA MCMC, et al., 2020; ROTHAN HA e BYRAREDDY SN, 2020; FRANCO JB, et al., 2020).

Aumenta com a idade, o risco de morte por COVID-19, visto que a maioria dos óbitos ocorrem em idosos com doenças crônicas (DE ALMEIDA HKS e SANTANA RF, 2020). Especialmente aqueles com comorbidades associadas, estando mais propensos a mortalidade adjuntas a infecção viral como a causada pelo SARS- Cov-2, pois apresentam maiores déficits no sistema imunológico, já os idosos que possuem diagnóstico anterior de doenças cardiovasculares, diabetes, hipertensão, e doenças cerebrovasculares estão sujeitos a estágios mais graves do COVID-19 (FLORES TG e LAMPERT MA, 2020; BEDFORD J, et al., 2020).

Devido a potencialidade de risco da população idosa ao contágio por COVID-19, esse grupo ganhou destaque; rapidamente foram direcionados ao isolamento e distanciamento social. Reforça-se nesta pandemia que além da pluralidade, diversidade e complexidade referentes ao envelhecimento humano, os idosos possuem características individuais e peculiares; diante disso não se pode coibir os fundamentos da prática e teoria gerontológicas, promovendo métodos diferenciais para a tomada de medidas eficazes para a proteção deste grupo de risco (DE ALMEIDA HKS e SANTANA RF, 2020).

$\mathrm{Na}$ busca em proporcionar atendimento odontológico seguro ao paciente e profissional, a odontologia desafia-se a realizar novas estratégias de biossegurança. Com isso o Conselho Federal de Odontologia (CFO) e a American Dental Association (ADA), orientam a realização apenas de procedimentos odontológicos de urgência e de emergência durante o período de pandemia (PEREIRA MCMC, et al., 2020).

Procedimentos considerados como urgentes estão relacionados a dores agudas, causadas por cáries extensas com envolvimento pulpar, pericoronarite, alveolite, abcessos ou infecção bacteriana envolvendo dor e edema, fraturas dentais, cimentação ou fixação de coroas ou próteses fixas onde sua falta de manutenção gere dor ou inflamação gengival e dos tecidos adjacentes, ajuste ou reparo de próteses removíveis que estejam causando dor ou prejudicando a função mastigatória, necroses orais que estejam causando dor ou com secreções purulentas, ajustes ou remoção do arco e de dispositivos ortodônticos que estiverem ulcerando a mucosa oral, trauma dentário com avulsão ou luxação do elemento dental (FRANCO JB, et al., 2020). 
Procedimentos de emergência como sangramentos não controlados, celulites ou infecções bacterianas difusas que causam edemas comprometendo as vias aéreas, traumatismos faciais, que também envolvam as vias aéreas do paciente (FRANCO JB, et al., 2020).

A higiene oral, mesmo em tempos de COVID-19, precisa ser compreendida como questão básica de saúde, considerando que o trato respiratório é a porta de entrada de infecção, boca (língua, dentes, gengivas e periodonto), faringe (garganta) e pulmões, onde os maiores agravos resultantes da infecção podem ocorrer (DA BAHIA GDE, 2020).

Portanto o principal objetivo é discorrer sobre a forma de atendimento odontológico em domicílio em tempos de pandemia causado pelo COVID-19 visando diminuir o risco de contaminação pelo SARS-CoV-2, onde nesta modalidade o idoso permanece no ambiente "livre de contaminação" e só recebe o profissional, para tratamentos de urgência e emergência, com os devidos cuidados.

\section{REVISÃO BIBLIOGRÁFICA}

Sendo um grande diferencial profissional, a odontologia praticada em domicílio, além de ser uma alternativa beneficente para a população idosa dependente e semi-dependente, geralmente também é bemvista por pacientes acometidos por doenças crônico-degenerativas no qual o enfoque gerontológico é seguido (BRAGA EC, et al., 2011).

Silva RMD, et al. (2020); retratam que no domicílio, o usuário pode sentir-se mais confiante para abordar questões que dificilmente levaria ao conhecimento do profissional na unidade básica de saúde (UBS) ou consultório odontológico, além de mais valorizado com a presença do profissional em sua casa. No entanto, é necessário que não se confunda vínculo, com dependência do usuário à presença do profissional de saúde. As práticas de saúde bucal precisam ser absolutas, orientadas para promover autonomia do usuário sobre o modo de viver a vida.

O diálogo entre o cirurgião dentista e o paciente é imprescindível para que o idoso, tenha segurança para fornecer todas as informações necessárias à um apropriado tratamento odontológico. O idoso que passa pelas transformações físicas associadas ao envelhecimento, deve ser abordado pelo cirurgião dentista como um paciente complexo, onde o profissional deve estar preparado para um diagnóstico bucal instantâneo, assim como para lidar com diferentes fatores sistêmicos que acometem o paciente (CUKIER MDOA, 2019).

É necessário a precisão de técnicas específicas de manejo e adequação profissional para que o procedimento seja efetivado de modo apropriado, onde a conduta técnica do procedimento clínico na prática odontológica domiciliar, realizada pelo odontólogo, não venha oferecer contestações, prevenindo e eliminando possíveis focos de sintomatologias dolorosas a fim de delinear e realizar procedimentos clínicos característicos e de mínima intervenção (BRAGA EC, et al., 2011; MIRANDA AF, et al.,2018).

A característica do atendimento odontológico é a proximidade face a face, entre odontólogo e paciente, então, medidas de biossegurança são fundamentais para evitar a transmissão de microrganismos, devido a exposição frequente a fluidos, saliva, sangue e produção de aerossóis, além de instrumentos cortantes manuais contaminados (TUÑAS ITDC, et al., 2020).

Para que seja possível oferecer um atendimento mais específico e de qualidade, a este grupo populacional de idosos que requer conforto e segurança, deve-se verificar a ampliação dessa atividade domiciliar, com um número maior de profissionais odontólogos especializados em Odontogeriatria, atendendo-os de forma mais individualizada e oferecendo maior bem-estar (SALES MVG, et al., 2017; CUKIER MDOA, 2019).

\section{Atendimento domiciliar em tempos de covid -19}

Com o decorrer dos anos, a população brasileira passou por mudanças demográficas, alterações de hábitos e de costumes (culturais). Além disso, em razão do aumento da expectativa de vida, a população idosa do país apresentou crescimento, ocasionando a necessidade de oferecer aos idosos qualidade de vida (SALES MVG, et al., 2017). 
Uma ferramenta relevante que pode ser utilizada por profissionais da área da saúde, assim como os cirurgiões dentistas, no momento atual é a realização de atendimento domiciliar a portadores de doenças crônicas e aos idosos, grupos com maior índice de mortalidade quando acometidos pela COVID-19 (DA CONSULTA A, et al., 2020).

Neste momento de pandemia causada pelo COVID-19 os cuidados que envolvem a manutenção da saúde bucal da população idosa, devem ser intensificados, tanto as que estão em Instituições de Longa Permanência (ILPI) quanto as que estão em suas residências (DA BAHIA GDE, 2020).

Ainda em momento pandêmico, o cirurgião dentista deve optar pela anamnese detalhada, mesmo diante de atendimentos de urgência, sendo esta, uma etapa que merece ser respeitada, onde deve conter informações sobre possíveis sintomas como alterações respiratórias, gastrointestinais, febre, desconforto geral nos últimos quatorze dias (DE OLIVEIRA JJM, et al., 2020).

Como justificativa para representantes do grupo de riscos para esta infecção por COVID-19, os idosos e os doentes crônicos, estão expostos a maiores números de injúrias e mortalidade, ligados ao próprio envelhecimento de forma fisiológica, apresentando problemas como a redução do metabolismo, diminuição da capacidade funcional, maior susceptibilidade às infecções, o que torna essencial a manutenção da saúde bucal (FLORES TG e LAMPERT MA, 2020; DA BAHIA GDE, 2020).

Parte integrante do corpo, a cavidade bucal é essencial sobre os aspectos fisiológicos e metabólicos, e sofre com o processo de envelhecimento, durante este processo surgem algumas alterações bucais como, periodontite, perda de dentes, gengivite, nota-se ainda, dores na articulação-temporo-mandibular (ATM), luxação devido à perda de dentes, próteses antigas com desgaste, próteses mal adaptadas e desgastes dos próprios dentes, são problemas de saúde oral notados ao envelhecer, gerando uma demanda maior em tratamentos restauradores extensos (MONTENEGRO FLB, et al., 2018; PRESA SL, 2014; DA ROCHA EF, et al., 2019).

O controle das infecções orais, compõem-se em procedimentos importantes no controle de distúrbios metabólicos e na prevenção de algumas doenças sistêmicas, como as pertinentes ao sistema respiratório, visto que os problemas bucais vão além dos limites dos dentes e da boca, onde surge a necessidade de incentivos em saúde por meio de programas governamentais de acesso ao tratamento odontológico para esse grupo populacional, devido aumento na expectativa de vida da população brasileira pois facilita o surgimento de doenças específicas da velhice, principalmente relacionadas à interação sistêmica com o aparelho estomatognático odontológico (DA ROCHA e MIRANDA AF, 2013; DA BAHIA GDE, 2020).

Alterações fisiológicas naturais podem gerar condições muitas vezes incontornáveis, e assim, os cuidados aos idosos, devem decorrer uma simples assistência para seu processo de progresso e cura, portanto os métodos preventivos são como eixo de qualquer intervenção que vise a saúde bucal na terceira idade (SIMÕES ACDA e CARVALHO DM, 2011; DOS SANTOS AA, et al., 2020). Infelizmente, os serviços públicos de saúde odontológica, possuem histórico de extrações e serviços de urgência, modulados por costume curativista, o que remete hoje em uma população idosa com altos índices de edentulismo, refletindo em uma saúde bucal precária (DUTRA CESV e SANCHEZ HF, 2015).

Visando a prevenção, promoção de saúde, recuperação, diagnóstico bucal, os idosos fragilizados devem receber atenção integral em ações odontológicas, com orientações e incentivos de hábitos saudáveis, estímulos de higiene pessoal e bucal com limpeza diária e regular dos dentes e próteses, e uso de dentifrícios com flúor para o fortalecimento da superfície dental, controle da dieta e saúde sistêmica (SIMÕES ACDA e CARVALHO DM, 2011; MIRANDA AF, et al., 2018; DOS SANTOS RJ, et al., 2018).

É importante os cirurgiões dentistas tomarem medidas rigorosas de proteção individual, e evitar ou minimizar procedimentos que possam gerar gotículas ou aerossóis, e ainda o uso de sugadores de saliva mais potentes, com jato de água com baixa potência, devem ainda estar atentos ao uso de equipamentos portáteis, observando a possível falta de ergonomia no novo ambiente de trabalho (CUKIER MDOA, 2019; MENG L, et al., 2020). 
Com a ausência de vacina contra o SARS-CoV-2 há a necessidade reforçada entre a população, em geral, a adoção das medidas de prevenção contra a infecção, preconizadas pela organização mundial da saúde (OMS), como realizar higiene das mãos, evitar ambientes fechados e aglomerações de pessoas. Para os profissionais da área da saúde, o uso dos óculos de proteção ou protetor facial (face shield), máscara cirúrgica/N95, avental, luva de procedimento e lavagem das mãos, devem ser utilizados para a prestação de assistência aos casos suspeitos ou confirmados de infecção por SARS-CoV-2 (BELASCO AGS e FONSECA CDD, 2020).

É importante a colocação de barreiras físicas entre os equipamentos, ainda uma proteção em toda a face, o cabelo e os braços do operador, sendo estas regiões bastante atingidas pelos respingos. Portanto, mesmo com suspensão do atendimento odontológico em algumas regiões, os atendimentos de urgência se fazem necessários a fim de que cirurgiões dentistas e pacientes estejam protegidos da transmissão do vírus SARSCoV-2 tornando essas medidas essenciais (FRANCO AG, et al., 2020).

\section{O papel da odontologia}

O acompanhamento odontológico, através de ações preventivas, oferece orientação sobre higiene bucal, próteses e diagnóstico precoce de possíveis lesões potencialmente cancerígenas, e sabemos do efeito negativo, no atraso no tratamento de tumores de cabeça e pescoço, muitas vezes encontrados na maxila e mandíbula (DE MELO RB, et al., 2020). Frente à esta situação, o auxílio domiciliar surge como uma opção para a promoção e manutenção da saúde, e para o enriquecimento na utilização dos recursos em saúde, a melhora da independência, a diminuição dos efeitos da incapacidade funcional ou da atividade da doença (VIEIRA RCF, et al., 2016).

Qualificado por compreender um serviço em saúde de atitude preventiva, curativa e educacional, realizado em pacientes idosos debilitados por estarem acamados ou impossibilitados de ir ao consultório, o atendimento odontológico domiciliar é considerado uma realidade de atuação profissional, em muitos países. Atendimento que visa a qualidade de vida, observando o paciente como um todo, constituindo saúde de maneira interdisciplinar (LEWIS A, et al., 2015; MIRANDA AF, et al., 2018).

A qualquer momento pode ocorrer uma emergência dental e pode agravar-se em pouco tempo necessitando de atendimento imediato, no entanto são necessários o uso de lençóis de borracha e sugadores de saliva mais potentes, assim como, o uso de óculos de proteção, protetores faciais (MENG L, et al., 2020). Sendo recomendado o adiamento de atendimento odontológico eletivo, dando prioridade à atendimentos de urgência, aos idosos que necessitam desse acompanhamento, devido à pandemia do COVID-19 os procedimentos devem seguir rígidos protocolos de biossegurança, os mesmos são revistos e remodelados com frequência pelas autoridades sanitárias (DA BAHIA GDE, 2020).

Uma inovação profissional que emerge é a odontologia domiciliar. Vertente desta nova prática odontológica, é o atendimento domiciliar e conveniente (CUKIER MDOA, 2019). Além de incitar o autocuidado através de ações preventivas e educativas, condutas clínicas particularizadas, a odontologia domiciliar fornece ao idoso dependente e ao semi-dependente uma melhor qualidade de vida (DOS SANTOS RJ, et al., 2018).

Os profissionais cirurgiões dentistas estão em constante exposição, durante o atendimento clínico odontológico, além dos aerossóis, saliva, sangue, instrumentos perfurocortantes contaminados durante o uso. Com isso, medidas de biossegurança, evitando a contaminação cruzada, são importantes para a proteção do odontólogo e do paciente (DE OLIVEIRA JJM, et al., 2020).

No entanto, os profissionais devem possuir conhecimentos sobre os artifícios da doença, suas manifestações orais e modos de transmissão (FERNANDES MDO, 2019). Devido às características dos procedimentos odontológicos, o risco de infecção cruzada entre cirurgião dentista e paciente é alto, pois o vírus SARS-CoV-2 facilmente pode infectar um indivíduo através do contato com secreções ou aerossóis (PELOSO RM, et al., 2020).

A telemedicina sugere que sempre que possível para o profissional da Odontologia, observar a necessidade real de atendimento, sem gerar danos à saúde do paciente e garantindo a segurança de todos, 
avaliando o bom senso entre a necessidade de intervenção e a menor exposição ao risco de contaminação (PEREIRA MCMC, et al., 2020).

É necessário o desenvolvimento de alguns protocolos para atendimento a domicílio, com organização na véspera do atendimento e um serviço de teleodontologia para averiguação sobre a saúde atual do paciente, utilização de medicamentos e estado clínico odontológico; é importante para o cirurgião dentista saber e orientar o paciente ou cuidador sobre a melhor condição para o paciente aguardar, remover excesso de objetos próximos para melhor locomoção do profissional e se necessário, seu assistente; manter o afastamento de crianças e animais domésticos; da mesma forma são separados instrumentais, que já devem estar esterilizados em ambiente adequado para isso, chegando prontos para uso na casa do paciente os equipamentos que virão a ser utilizados no procedimento a ser realizado; anestésicos, soro, material para descarte de perfurocortantes, sem esquecer dos Equipamentos de Proteção Individual (EPI) (CURY MDS, 2012).

Neste momento de pandemia é necessário manter o cuidado com a biossegurança para o atendimento domiciliar, pois quem irá entrar em um ambiente que poderá estar contaminado é o cirurgião dentista e não o paciente, devendo o odontólogo manter um ritual com a preparação de biossegurança antes de adentrar a residência do paciente, devendo já estar previamente paramentado, para não correr o risco de contaminar o domicílio e o idoso, assim como os familiares ou cuidadores residentes, devem estar com máscaras, e higienizar as mãos com frequência (CURY MDS, 2012). Percebe-se que assim como na década dos anos oitenta, após o surgimento da Aids, faz-se necessário mudanças comportamentais, no âmbito da saúde. Essas alterações para maior segurança dos profissionais da saúde fazem parte de uma evolução para todos (FERNANDES MDO, 2019).

O protocolo de paramentação para atendimento aos pacientes com suspeita de COVID-19 quando o procedimento não puder ser adiado inclui: higienizar as mãos, calçar o primeiro par de luvas, colocar o avental impermeável (prender no pescoço e depois na lateral), colocar a máscara cirúrgica sobre a máscara N95 ou PFF2, colocar os óculos de proteção, colocar gorro (cobrindo as orelhas), calçar o segundo par de luvas cobrindo parte das mangas do avental (FRANCO ABG, et al., 2020).

Sendo o protocolo de desparamentação: higienizar luva externa, remover o avental e a luva externa simultaneamente, enrolando-os sempre pegando na parte interna do avental, descartar em saco de lixo usado apenas para descarte de EPIs, retirar o gorro de trás para frente, e descartá-lo no saco de lixo, retirar o óculos de proteção e colocar em saco plástico, retirar a máscara cirúrgica e descartar no saco de lixo, higienizar a luva interna, e retirar descantando no saco de lixo, higienizar as mãos; após a realização do procedimento como o manuseio de pasta, caneta, carimbo, higienizar as mãos com friccionamento de álcool 70\%.O recebimento de honorários para atendimentos particulares deve ser feito preferencialmente por transferência bancária, ou similar (FRANCO ABG, et al., 2020).

\section{A prestação de serviço em domicílio no Brasil}

No período de pós-guerra nos Estados Unidos, devido ao aumento de pessoas com necessidade de atendimento e poucas vagas nos hospitais da época, surgiu uma modalidade que vem sendo praticada até hoje, o Cuidado em Casa (Home Care). Sendo organizado inicialmente no ano de 1796. Já no Brasil o serviço de atendimento Home Care tem seus primeiros registros no ano de 1968, restritos a atendimento de vigilância epidemiológica materno-infantil tendo sua organização a nível nacional em 1990 após aprovada a Lei 8.080 que entre outras, regulamenta a assistência domiciliar no Brasil; a Anvisa (Agência Nacional de Vigilância Sanitária) em 2006 publicou a RDC n‥ 11, que estipula regras para o funcionamento de serviços de saúde que prestam atendimento domiciliar, que a partir disso começam a ser melhor estruturados, com normas para o funcionamento, pois passam a ser fiscalizados (BRAGA PP, et al., 2016; LISBOA E, 2016; BARBOSA E, 2017; REIS LRA, et al., 2019).

Assim como, a movimentação financeira decorrida do negócio de assistência domiciliar na economia mundial, também são multiplicadas as oportunidades para empreendedores e geração de emprego para a população local, com o aumento da população idosa e o interesse pela qualidade no cuidado dos idosos, pois 
aumenta a estimativa de vida, mas a saúde continua carente de cuidados onde a qualidade de vida será sempre um desafio na terceira idade (LISBOA E, 2016).

Ações empreendedoras na área da saúde fazem-se necessárias diante do cenário atual, mostrando inovações nas ações e oferecendo atendimento especializados, a partir de estratégias dinâmicas de forma a solucionar problemas sociais, por meio de práticas contemporâneas e projetos (REIS LRA, et al., 2019). Um estudo levantado e divulgado em 2018 pela Federação dos Hospitais, Clínicas e Laboratórios do Estado de São Paulo (FEHOESP) mostrou que houve um aumento de 35\% no serviço de Home Care no Brasil (LISBOA E, 2016).

Além da odontologia vários segmentos já oferecem atendimento Home Care, como profissionais terapeutas, fisioterapeutas, médicos, fonoaudiólogos, dentre outros, com destaque para a área da enfermagem que representa um grande número de oferta de trabalho para atendimento em domicílio, sendo na prática da atividade pelo sistema público ou privado (REIS LRA, et al., 2019).

Ainda neste contexto, mostra-se a importância da formação e capacitação dos profissionais que atendem pacientes dependentes e semi-dependentes em domicílio, visto que as necessidades destes pacientes são específicas, onde 0 cuidador deve estar bem-preparado para atender as exigências que esta modalidade determina (BRAGA PP, et al., 2016; CASTRO EABD, et al., 2018).

A modalidade de atendimento Home Care, demonstra redução de gastos com despesas hospitalares para pacientes que necessitam de internação muito longa, e cuidados diários, seja por doenças crônicas, dificuldades de locomoção, doenças terminais, paciente pós cirúrgicos, idosos, pacientes com necessidades especiais, o que gera a necessidade de equipes multidisciplinares capacitadas para este tipo de procedimento, além de oferecer serviços mais humanizados com o conforto do lar do paciente (BRAGA PP, et al., 2016; REIS LRA, et al., 2019).

\section{CONSIDERAÇÕES FINAIS}

Pôde ser observado que a necessidade de atendimento especializado ao idoso, têm crescido com o passar dos anos, por ser uma população que está em busca de qualidade de vida sendo acometida por várias limitações e problemas de saúde também decorrentes do envelhecimento. A modalidade Home Care, já faz parte dos atendimentos em vários países há muitos anos, e no Brasil vem crescendo significativamente ao longo do tempo. O cuidado em relação a contaminação por COVID-19 deve ser observado pelos profissionais da saúde, sendo o atendimento domiciliar ao idoso uma proposta que precisa ser visualizada como uma medida eficaz, preventiva, multiprofissional, principalmente nos casos de emergência, ou mesmo em casos onde o idoso não possa comparecer em consultório odontológico, visando também, sua perspectiva e qualidade de vida.

\section{REFERÊNCIAS}

1. BARBOSA E. Profissionais da saúde \& home care. Thieme Revinter Publicações LTDA, 2017; 13.

2. BEDFORD J. COVID-19: towards controlling of a pandemic. The Lancet, 2020; 395(10229): 1015-1018.

3. BELASCO AGS, FONSECA CDD. Coronavirus 2020. Revista Brasileira de Enfermagem, 2020; 73(2).

4. BRAGA EC, et al. Intervenção odontológica domiciliar em paciente idoso cego institucionalizado, Relato de caso. Revista Longeviver, 2011; 15.

5. BRAGA PP, et al. Oferta e demanda na atenção domiciliar em saúde. Ciência \& Saúde Coletiva, 2016; 21 : 903-912.

6. CASTRO EABD, et al. Organização da atenção domiciliar com o Programa Melhor em Casa. Revista Gaúcha de Enfermagem, 2018; 39.

7. CUKIER MDOA. Desafios da assistência odontológica domiciliar aos idosos, 2019.

8. CURY MDS. Sugestão de protocolos para atendimentos domiciliários em pacientes idosos, 2012.

9. DA BAHIA GDE. Recomendações para o cuidado da saúde bucal das pessoas idosas, 2020.

10. DA CONSULTA $A$, et al. Orientações para atendimento domiciliar a idosos por geriatras, especialistas em gerontologia e outros profissionais da saúde, 2020.

11. DE ALMEIDA HKS, SANTANA RF. Saúde do idoso em tempos de pandemia COVID-19. Cogitare Enfermagem, 2020; 25. 
12. TUÑAS ITDC, et al. Doença pelo Coronavírus 2019 (COVID-19): Uma abordagem preventiva para Odontologia. Rev. bras. odontol, 2020; 1-6.

13. DE MELO RB, et al. COVID-19 and the Invisible Damage. Acta Médica Portuguesa, 2020; 33(13).

14. DE OLIVEIRA JJM, et al. O impacto do coronavírus (covid-19) na prática odontológica: desafios e métodos de prevenção. Revista Eletrônica Acervo Saúde, 2020; 46: e3487.

15. DOS SANTOS AA, et al. Higiene e saúde bucal em idosos na atenção primária: uma revisão sistemática. Revista Eletrônica Acervo Saúde, 2020; 44: e2673-e2673.

16. DOS SANTOS RJ, et al. Odontologia domiciliar como parte integrante da assistência em saúde de idosos frágeis. Revista Longeviver, 2018.

17. DUTRA CESV, SANCHEZ HF. Organização da atenção à saúde bucal prestada ao idoso nas equipes de saúde bucal da Estratégia Saúde da Família. Revista Brasileira de Geriatria e Gerontologia, 2015; 18(1), 179-188.

18. FERNANDES MDO. Atitudes de Cirurgiões-dentistas da cidade de Natal no atendimento a pacientes portadores do HIV/AIDS. Bachelor's thesis, Universidade Federal do Rio Grande do Norte; 2019.

19. FLORES TG, LAMPERT MA. Por que idosos são mais propensos a eventos adversos com a infecção por covid-19. Monografia [pós-graduação em gerontologia] Universidade Federal de Santa Maria, Santa Maria-RS-Brasil; 2020.

20. FRANCO ABG, et al. Atendimento odontológico em UTI's na presença de Covid 19. InterAmerican Journal of Medicine and Health, 2020; 3: e20200304.

21. FRANCO AG, et. al. Importância da conduta do cirurgião-dentista frente à contenção e prevenção do Covid-19. InterAmerican Journal of Medicine and Health, 2020; 3.

22. FRANCO JB, et al. Cuidados Odontológicos na era do COVID-19: recomendações para procedimentos odontológicos e profissionais. Rev. Assoc. Paul. Cir. Dent, 2020; 74(1): 18-21.

23. LEWIS A, et al. Improving the oral health of frail and functionally dependent elderly, 2015. Australian dental journal, 2015; 60: 95-105.

24. LISBOA E. Modelos de negócio de assistência domiciliar com foco em idosos nos Estados Unidos e no Brasil: uma análise comparativa, 2016.

25. MENG L, et al. Coronavirus disease 2019 (COVID-19): emerging and future challenges for dental and oral medicine. Journal of Dental Research, 2020; 99(5): 481-487.

26. MIRANDA AF, et al. Odontologia Domiciliar como Parte Integrante da Assistência em Saúde de Idosos Frágeis. Revista Ciências e Odontologia, 2018; 2(2): 33-38.

27. MONTENEGRO FLB, et al. Atenção odontológica para idosos em unidades de internação. Revista Longeviver, 2018; 7.

28. PELOSO RM, et al. How does the quarantine resulting from COVID-19 impact dental appointments and patient anxiety levels?. Brazilian oral research, 2020; 34.

29. PEREIRA MCMC, et al. Desafios do atendimento odontológico ao paciente oncológico em tempo de COVID-19 Challenges of dental care to oncological patients in times of COVID-19, 2020.

30. PRESA SL. Saúde bucal na terceira idade. Revista Uningá, 2014; 39(1).

31. REIS LRA, et al. Assistência ao paciente idoso prestada pelo enfermeiro na home care: uma prática empreendedora. Revista de Saúde ReAGES, 2019; 1(4): 28-30.

32. ROCHA DA, MIRANDA AF. Atendimento odontológico domiciliar aos idosos: uma necessidade na prática multidisciplinar em saúde: revisão de literatura. Revista Brasileira de Geriatria e Gerontologia, 2013; 16(1): 181-189.

33. DA ROCHA EF, et al. Envelhecimento humano e desenvolvimento da doença periodontal. Revista Eletrônica Acervo Saúde, 2019; (26): e775-e775.

34. ROTHAN HA, BYRAREDDY SN. The epidemiology and pathogenesis of coronavirus disease (COVID-19) outbreak. Journal of autoimmunity, 2020; 102433.

35. SALES MVG, et al. Condições de saúde bucal do idoso no Brasil: uma revisão de literatura. Arch Health Invest, 2017; 6(3): $120-4$.

36. SILVA RMD, et al. Atuação da equipe de saúde bucal na atenção domiciliar na Estratégia Saúde da Família: uma revisão integrativa. Ciência \& Saúde Coletiva, 2020; 25: 2259-2270.

37. SIMÕES ACDA, CARVALHO DM. A realidade da saúde bucal do idoso no Sudeste brasileiro. Ciência \& Saúde Coletiva, 2011; 16: 2975-2982.

38. VIEIRA RCF, et al. Atendimento odontológico domiciliar ao idoso e a necessidade de tratamento endodôntico. Revista Brasileira de Odontologia, 2016; 73(1): 9. 